\title{
Significado Relacional: Una propuesta de integración entre la tradicional observación de bebés y la semiótica
}

\author{
Patricia Cordella ${ }^{1}$, Andrea Dainesi ${ }^{2}$, Stefanella Costa-Cordella ${ }^{3}$
}

Introducción: El contexto socioemocional en la infancia es un factor esencial para la configuración neuro psíquica. La observación de la relación madre-lactante permite evaluar su funcionamiento y además aprender acerca de cómo opera la mente preverbal. Objetivos: Presentar un modelo de comprensión relacional madreinfante que integre el modelo de la tradicional observación de bebés $(\mathrm{OB})$ junto con un proceso de generación de sentido relacional. Método: Se presentan 14 semanas de observación de una niña entre la semana 8 y 24 que incluyen la participación del observador como instrumento de resonancia afectiva. Resultado: Se muestran los pasos del procedimiento que genera sentido a partir de los eventos interaccionales madrelactante-observador. Se propone un conjunto básico de conceptos dinámicos necesarios para la construcción de este modelo de comprensión relacional. Conclusiones: La generación de sentido a partir de interacciones requiere de un procedimiento que integre: la observación interaccional; la experiencia del observador participante durante dicha observación; los referentes conceptuales elegidos. La comprensión de las dinámicas relacionales origina un modelo de construcción de sentido relacional. Este modelo utiliza las categorías perceptibles de: procesos somáticos; arousal; conductas y afectos.

Palabras clave: Observación de bebés; semiótica; relacional; desarrollo infantil; psicoterapia; significado.

Introduction: The socio-emotional context of infant development is a crucial factor in the neuropsychic conformation. Direct observations on mother-infant interactions allow an assesment of its functioning as well as gaining further understanding of how the preverbal mind operates. Aim: To propose a comprehensive model of analyses of the mother-infant relationship that integrates the method of infant observation with a process of relational "meaning construction". Method: Observations ( 14 weeks) on an infant girl (from $4^{\text {th }}$ to $24^{\text {th }}$ week of age) are presented, including the observer as an instrument of affective echoing. Results: The relational "meaning construction" is described in each step of the process originating from the relational events which involve the infant, the mother and the observer. A combination of essential psychodynamic concepts will be adopted to formulate a comprehensive relational model. Conclusions: Relational meaning construction from the dyadic interactions requires a process that combines: 1 ) the direct observations of the interactions; 2 ) the affective experience of the observer; 3) an underlying conceptual psychodynamic framework. The understanding of the interactional processes generates a potentially innovative model on "relational meaning construction". Such a model is supported by the categories: a) somatic processes, b) arousal, c) conducts and affects

Key Words: Infant observations; semiotic; relational; psychotherapy; meaning

English Title: Relational Meaning Construction: a proposal of integration between semiotics and classical infant observation.

\section{Cita bibliográfica / Reference citation:}

Cordella, P., Dainesi, A. y Costa-Cordella, S. (2019). Significado Relacional: Una propuesta de integración entre la tradicional observación de bebés y la semiótica. Clínica e Investigación Relacional, 13 (2): 389-407. [ISSN 1988-2939] [Recuperado de www.ceir.info] DOI: 10.21110/19882939.2019.130205

\footnotetext{
${ }^{1}$ M.D Ph.D Departamento de Psiquiatría, Facultad de Medicina, Pontificia Universidad Católica de Chile. La correspondencia de este trabajo debe ser dirigida a: mpcordella1@gmail.com

2 Psychologist Ph.D Anna Freud Centre London, Research Department of Clinical, Educational and Health Psychology, University College London

3 Psychologist Ph.D Research Department of Clinical, Educational and Health Psychology, University College London; Facultad de Psicologia, Universidad Diego Portales.
} 


\section{Introducción}

En la clínica de lactantes hay múltiples oportunidades para observar la interacción madrebebé y comprender la forma en la que ambos construyen un sistema relacional. (Hofer ,1984)

Este contexto socioemocional le ofrece al lactante regularidades y modos de estar con otros, especialmente sus cuidadores. Lo dispone al acoplamiento y aprendizaje haciendo que las estructuras neurológicas se acomoden y adapten durante el desarrollo (Tottenham, 2015). Repetir genera estimulación de conexiones, patrones sensoriomotores que organizan el tejido cerebral. (Pratt et al. 2017). Por un lado, los estados de alarma y por otros los de motivación como los de arraigo a la realidad van organizando el sistema nervioso del infante, (Tost, Champagne \& Meyer-Lindenberg, 2015) lo va personalizando (Winnicott, 1962) y son la base del self.

Una figura reguladora que lea y enseñe a leer los estados mentales propios y ajenos (Fonagy \& Allison, 2012) que acepte, respete y resuene con el lactante proporcionaría un bienestar psíquico y somático suficiente para un funcionamiento neuro psíquico adecuado. Esta regulación (Schore,2018), no solo debe organizar la experiencia sino hacerla articulable al grupo de pertenencia (Fonagy, 2018).

La organización de las neuro estructuras en desarrollo se apoya y ancla en zonas corporales cargadas de sensibilidad y expresión cortical sensorial y motora. (Freud, 1905) Una amplia red de receptores asegura la aferencia del ambiente. Las sensoriales: táctiles, olfativos, visuales, auditivos y gustativos; las interoceptivas de las mucosas y las propioceptivas de músculos y huesos. La posición del cuerpo y los movimientos en el espacio se suman a la amplia representación cortical, sensorio- motora. (Riva, 2018) Zonas como la boca, el ano y los genitales están cargadas de receptores que facilitan la comunicación con el ambiente/otro. (Ismail,Fatemi, \& Johnston, 2017).La integración neuro psíquica es un proceso largo, de múltiples entradas y con altas probabilidades de micro o macro fallas. (Taylor, 2018) La arquitectura cerebral humana requiere de un vínculo afectivo. (Sravanti, 2017). donde actuar y sentir sean intercambiados entre un humano naciente y otro adelantado. La integración del tejido nervioso desde el tronco cerebral, cerebro medio y corteza prefrontal se favorece con un ambiente facilitador de la maduración (Winnicott,93) puede afectarse con experiencias socioemocionales traumáticas como la violencia o el abandono (Perry,1995)

Aprender a observar relaciones en la formación de especialistas en psicoterapia y desarrollo ha sido una preocupación hace más de cincuenta años. (Freud, 1976). Un observador 
presente, atento y dispuesto a participar del sistema relacional madre-bebé puede recoger información valiosa sobre el contexto socioemocional y el curso del desarrollo neuro psíquico del lactante. (Beebe et al. ,2010).

La observación de relaciones se entrena a través de diversas formas de aprendizaje. La más antigua es la observación de bebés (OB) practicada en la tradición psicoanalítica. Tiene su origen en autores como Anna Freud (1958), Melanie Klein (1952), Aguayo, Salomonsson (2017), Wilfred Bion (1982), Donald Winnicot (1941) y más tarde Esther Bick (1964) quien la protocolizó como es practicada hoy. El inicio de este entrenamiento estuvo marcado por la reconstrucción de la experiencia infantil directa, lo que antes solo podía hacerse con análisis retrospectivo en el análisis de pacientes adultos. (Zaphiriou et al., 2016). Estos autores se interesaron en observar, en tiempo presente, el desarrollo mental infantil y las manifestaciones del niño/a, especialmente en presencia o ausencia de la madre. Así tuvieron acceso a la de construcción del psiquismo. Se propusieron estudiar: los comportamientos, las funciones de esos comportamientos y las defensas contra las angustias cotidianas. (Symington, 2018). Como el lactante es pre-verbal, acuñaron conceptos que pudieran describir la actividad mental que se realiza sin palabras. Actividad que continúa toda la vida de forma inconsciente.

La OB es considerada una herramienta valiosa en el entrenamiento de quienes trabajan con niños ( $\mathrm{Ng}$ et al., 2017). La consigna es observar, en su casa, un niño/a y su madre durante 6 o 12 meses, ojalá desde el nacimiento; anotar lo visto y vivido para elaborarlo en un grupo de supervisión. Estos tres pasos (observar- registrar-mostrar/ discutir en grupo) consiguen en la formación del Observador: a. una disciplina de observación relacional; b. un aumento en la capacidad empática del Observador; c. un patrón de acomodo al ritmo y formas relacionales; d. la ganancia de una posición neutra (curiosa, pero no invasiva ni distante) desde donde mirar, sentir y pensar una relación y e. contacto con sus propias vivencias pre-verbales y posibilidad de reelaborarlas.

Se trata de una técnica de campo que registra el micro-proceso relacional madre-hijo/a basada en que: 1. El Observador enfoca las escenas que selecciona (con criterios arbitrarios y no siempre conscientes) tomando notas tanto de la percepción sensorial externa como de la propia (interna); 2. La observación es un proceso que tiene una duración preestablecida y se realiza una vez por semana; 3. La madre acepta participar en esta actividad sabiendo que el Observador no es una ayuda, no es un consejero, no está diagnosticando y no dará un informe final.

La OB es "una presencia amistosa, no-intrusiva e interesada que pone atención a los pensamientos y sensaciones de los miembros de la familia". Rustin (2014). La posición que 
permite recibir la información de mentes próximas operando en el CV es una mente "sin memoria, sin deseo, sin comprensión". Bion (1984).

La OB favorece la sensibilidad, agudeza, refinamiento y precisión de la observación de los fenómenos relacionales emergentes (Rustin, 2014). El observador entrenado ampliará su receptividad perceptiva, empática e inconsciente (Izzedin- Bouquet, 2009). Este entrenamiento permite pesquisar patrones de riesgo para el desarrollo psíquico y somático infantil (Minjollet et al., 2018) y considerar derivaciones e intervenciones. (Hutchon et al., 2019).

La semiótica se ocupa del proceso de construcción, uso y funcionamiento de los signos. Los signos permiten comunicar y otorgan sentido a lo percibido. Un signo es algo que para alguien representa algo $(E c o, 1988)$. Es el modo de participar de una mente común, dinámica en constante reinvención.

Una percepción se hace 'presente en la mente quien, al ligarla con alguna dimensión coherente, lo trasforma en un objeto comunicable (por ej. Palabra, sueño, imagen). Este objeto es a su vez una percepción que se hace presente en una o más mentes. Un signo se apoya en una percepción de realidad, pero no la construye, es necesario ligar este indicio a un referente que lo integre a un universo con sentido. Cada signo se liga a otro signo que lo refiere a otro signo. (Pierce, 1931). Este peregrinaje en busca de sentido, abre y cierra permanentemente los significados de las cosas.

El clínico es un observador relacional que organiza lo perceptible para hacerlo significar en función del cambio. La interpretación o resignificación de lo percibido acomodaría el equilibrio emocional perdido y restauraría el bienestar.

El observador relacional aprende a hacer semiología relacional, es decir, a identificar los fenómenos relevantes de una relación humana en tercera y primera persona: ve y siente lo que allí ocurre en una experiencia directa, en tiempo presente, no a través de videos o test. El observador está presente en la escena. No debe dar indicaciones ni hacer juicios. No hace, observa. En un segundo tiempo el observador busca y articula ciertos referentes teóricos que selecciona para ligarlos a lo observado. Se construye, así como un receptor semiótico (Cordella, 2014) que le da sentido a lo observado. El observador al observar en tiempo cero, no es un clínico que busca diagnósticos y soluciones. Tampoco es un investigador que selecciona variables para sus hipótesis. Es un curioso que aprende de aquello que observa: vacío de prejuicios, sin deseo y sin temor. 
En este artículo se han seleccionado conceptos teóricos del universo psicoanalítico, validados por su uso en psicología y otros campos relativos a fenómenos humanos. Estos son capaces de describir fenómenos comunicacionales inconscientes, tomando en cuenta que el infante se encuentra en un momento de desarrollo psíquico primigenio pre-verbal.

El objetivo de este trabajo es proponer un modelo de comprensión de la relación madreinfante basado en la clásica observación de bebés mostrando los pasos de generación de sentido a partir de la observación interaccional y la articulación con una serie de conceptos dinámicos que facilitan la semiología relacional.

\section{Material y Método}

a. Descripción del caso: Adele es la menor de 4 hermanos adolescentes. Vive con padre y madre (Lyn) en un país donde son extranjeros. El caso presentado ha sido organizado en 8 observaciones durante 14 semanas a partir de la semana 8 de vida de Adele. Las observaciones fueron seleccionadas a partir de los registros tomados por el mismo Observador por tratarse de eventos relacionales con potencial significado.

b. El material que ilustra la OB fue recogido por uno de los investigadores (A.D) durante 16 semanas en un contexto formativo en el Anna Freud Center, Londres, durante el año 2018. Se trata de una lactante (Adele) y su madre (Lyn) que son observadas desde la semana 8 a la 24 en ) en 9 registros .Los protocolos éticos con protección de la identidad de ambo son aprobados. Lyn acepta con la expectativa de sumar al desarrollo de su bebé, aunque se le explicita que no se realizará intervención alguna con ella ni su hija.

El método utilizado en la OB es el habitual: se recogen notas de las acciones de la diada y de los sentimientos, acciones o emociones del observador.

\section{Resultados}

Se presentan en las Tablas 1,2,3, y 4 las diferentes trasformaciones que le van dando sentido a lo observado.

La Tabla No 1 muestra la totalidad de los registros realizados. De estos registros se selecciona una observación. (numero 2) 
Tabla $\mathrm{N}^{\circ} 1$ : Registro de observaciones de la interacción madre-hija desde 8 a 24 semanas de vida.

\begin{tabular}{|c|c|c|}
\hline $\begin{array}{l}\text { OBSER } \\
\text { VACIÓN }\end{array}$ & $\begin{array}{l}\text { EDAD EN } \\
\text { SEMANAS } \\
\text { DE ADELE }\end{array}$ & REGISTRO DE OBSERVACIÓN \\
\hline 0 & $8 \mathrm{~s}$ & $\begin{array}{l}\text { En esta primera reunión, destinada a establecer las normas de nuestros encuentros, Lyn me dejó } \\
\text { solo con la bebé (Adele) que dormía y volvió cuando le tuve que avisar que lloraba y recordarle } \\
\text { que no venía a cuidar a Adele. Lyn aceptó nuevamente esta condición. Dejo la casa con la } \\
\text { sensación de no haber sido comprendido. Me siento confuso y desatendido. }\end{array}$ \\
\hline 1 & 95 & $\begin{array}{l}\text { Adele se encontraba durmiendo en su cuna, otra vez. Lyn, en pijama, se retira inmediatamente } \\
\text { dejándome con Adele en una sala muy desordenada. La mayor parte de esta sesión transcurrió } \\
\text { observando como dormía Adele calmada y relajada. Al despertar, comenzó a llorar moviendo } \\
\text { piernas y brazos frenéticamente, por algunos minutos. Me sentí cada vez más nervioso. Estaba } \\
\text { muy preocupado sintiendo que tal vez Lyn no podía escuchar a Adele, ya que no venía. Me } \\
\text { preocupé tanto que perdíel control sobre los límites de mi propio rol: me paré y fui a la cocina a } \\
\text { buscar a Lyn para decirle que Adele estaba llorando. Lyn respondió que ella estaba ocupada y } \\
\text { que si podía yo por favor mecer un poco la cuna para que la bebé se calmara. Eso hice, por unos } \\
\text { minutos. Adele se calmó y se volvió a dormir. En ese momento, mi sensación fue de } \\
\text { desorientación y angustia y la pude recién elaborar en el grupo de discusión del curso académico. }\end{array}$ \\
\hline 2 & $10 \mathrm{~s}$ & $\begin{array}{l}\text { Adele se despierta y sus ojos comienzan a ponerse cada vez más grandes. Lentamente mueve su } \\
\text { cabeza, luego detiene su mirada en un juguete que cuelga encima de la cuna. Mueve todo su } \\
\text { cuerpo, y sus brazos los mueve en movimientos circulares. Repentinamente, sale leche de su } \\
\text { boca, como una regurgitación. Comienza a llorar con creciente angustia y esta vez comienza a } \\
\text { sacudir también las piernas. Esto continúa por unos minutos. Siento rabia, impotencia y angustia } \\
\text { en esta demora. No sabía bien que hacer. Tenía la teoría, pero no estaba seguro. Voy donde Lyn } \\
\text { y le aviso que Adele llora y me devuelvo a mi silla esperando que sea Lyn quien venga a calmar a } \\
\text { Adele. Lyn llega y se sienta detrás de la cuna. Mueve la cuna con sus pies y le dice unas palabras } \\
\text { a Adele en su idioma nativo. Adele deja de llorar inmediatamente y, con los ojos bien abiertos, } \\
\text { fija su mirada en un juguete que cuelga y que se está moviendo al mismo tiempo que la cuna. Le } \\
\text { informo a Lyn acerca de la regurgitación. Ella me responde que se pregunta por qué Adele estará } \\
\text { llorando si es que ya comió y durmió. }\end{array}$ \\
\hline 3 & $11 \mathrm{~s}$ & $\begin{array}{l}\text { Adele esta tendida en las piernas de Lyn. Su cabeza está doblada hacia atrás, y mira hacia arriba } \\
\text { mientras toma leche de un biberón sostenido por su madre. Lyn no la está tocando ni mirándola. } \\
\text { Lyn está en el teléfono y al mismo tiempo lee unas cartas por encima. Esta dinámica continúa por } \\
\text { varios minutos, mientras Adele toma leche inmóvil, mueve su mirada entre el techo y entre el } \\
\text { lado opuesto a aquel donde está su madre. Repentinamente, Lyn mientras habla por teléfono y } \\
\text { lo pone a cargar, cubre la cara de Adele con la mano con la que está sosteniendo el biberón. En } \\
\text { aquel momento, Adele mueve su cabeza y agita sus piernas bruscamente, despegándose del } \\
\text { biberón. Lyn trata de ponerle el biberón de nuevo adentro de la boca. Adele gira su cara retirando } \\
\text { la boca del biberón y estira sus brazos hacia su madre, mirándola. Luego de varios intentos, Lyn } \\
\text { logra insertar nuevamente el biberón en la boca de Adele. Siento que la madre me provoca, } \\
\text { exhibiendo este modo desconectado, indiferente a las necesidades de su hija. }\end{array}$ \\
\hline 4 & $13 \mathrm{~s}$ & $\begin{array}{l}\text { Lyn está meciendo la cuna de Adele. Mientras mueve su cabeza para explorar el ambiente, Adele } \\
\text { me mira por un momento y luego redirige la mirada rápidamente hacia su madre, quien está } \\
\text { hablando por teléfono y no se la devuelve. Unos instantes después, su mirada vuelve hacia mí. } \\
\text { Esta vez se queda ahí por más largo tiempo. Fija sus ojos en los míos por momentos con su boca } \\
\text { abierta pero inmóvil. Me siento muy bien en sintonía con ella. Conectado e incluido. Luego gira su } \\
\text { mirada hacia su madre, agitando los brazos y moviendo su cabeza hacia arriba y hacia abajo. } \\
\text { Esta dinámica se repite un par de veces. Luego, Lyn toma un juguete y lo agita en frente de la } \\
\text { cara de Adele, sin mirarla y continúa con su llamada telefónica. Adele inmediatamente dirige su } \\
\text { atención hacia el juguete. Me siento frustrado con la madre, pero conectado y unido con Adele lo } \\
\text { que alivianaba mi dificultad para mantenerme en mi rol. }\end{array}$ \\
\hline
\end{tabular}




\begin{tabular}{|c|c|c|}
\hline 5 & $14 \mathrm{~S}$ & $\begin{array}{l}\text { Lyn está cambiando el pañal de Adele. La toma en brazos y la acuesta sobre la cama. Adele } \\
\text { parece calmada mientras la cambian: en algunos momentos mueve sus piernas con fuerza y } \\
\text { excitación, sonriendo; en algunos momentos deja que su madre guie sus movimientos y ríe. } \\
\text { Durante estas interacciones, Lyn va comentando cada movimiento y acción:" Oh, ite gusta que } \\
\text { te esparza la crema" "buena niña!" Adele ríe y agita sus brazos excitadamente. Mientras Lyn } \\
\text { esparce la crema en su cuerpo, ambas se miran a los ojos intensamente. Me sentí tranquilo, } \\
\text { sereno y fue placentero. En armonía con la diada, confortable en mi rol. }\end{array}$ \\
\hline 6 & $15 \mathrm{~s}$ & $\begin{array}{l}\text { Lyn le echa crema a Adele en la cara. Adele se estresa y comienza a llorar abriendo los ojos y } \\
\text { agitando la cabeza. Lyn dice "disculpa" y le pone el biberón en su boca. "Quieres leche?". Pero } \\
\text { Adele trata de sacar su cara de la mamadera, agotando sus brazos abiertos, produciendo sonidos } \\
\text { agudos. Lyn dice "Ah, no, tú quieres leche" la toma en brazos y la pone en dirección hacia mí. } \\
\text { Cuando Adele me mira, yo la miro de vuelta. Lyn comenta que deliberadamente elige usar el } \\
\text { biberón más frecuentemente que su pecho porque "ayuda a los bebés a ser más independientes". } \\
\text { Deja de llorar, pone sus ojos grandes y mira de vuelta a su madre, quien le toma las primas en un } \\
\text { modo juguetón. Luego, Lyn toma un poco de crema y se la echa de nuevo en la cara a Adele, } \\
\text { quien, a este punto comienza a llorar fuerte. Lyn le dice "Viste! Tenías hambre" Adele sigue } \\
\text { llorando y me mira a mí de nuevo. Siento rabia por haber perdido esa armonía anterior. Me sentí } \\
\text { víctima de los imprevisible de la madre. La rabia y la frustración me empujaban a interveniry salir } \\
\text { de mi rol impotencia. }\end{array}$ \\
\hline 7 & $21 \mathrm{~S}$ & $\begin{array}{l}\text { Adele está en su cuna, con su cara apuntando hacia la televisión. Lyn se sienta en un sillón detrás } \\
\text { de la cuna y pone la mamadera cerca de la boca del bebé, mientras lee el periódico cuando el bebé } \\
\text { mira el biberón encima de su cabeza, comienza a tratar de tomarla con las dos manos. Luego, } \\
\text { mientras toma la eche, Adele hace unos sonidos fuertes y mueve sus piernas. Mientras toma la } \\
\text { leche se va agitando y comienza a empujar el biberón, dando vuelta su cabeza hacia otro lado. } \\
\text { Luego de unos minutos, Lyn mira hacia la niña y le dice: "tú no necesitas leche" y retira el biberón } \\
\text { de la boca de Adele. Adele fija sus ojos en la televisión, agitando sus piernas con fuerza. Mientras } \\
\text { hace esto, ella comienza a succionar sus manos. Deja de mirar la televisión y comienza a observar } \\
\text { sus dedos en un modo exploratorio. Luego de mirar fijamente sus manos por varios minutos, mira } \\
\text { hacia la televisión de nuevo y pone ambas manos adentro de su boca. Sentí que Adele estaba } \\
\text { más grande, que buscaba su autonomía concentrándose en un mundo propio, absorta y } \\
\text { concentrada en él. Al parecer su desarrollo era adecuado con algunos resabios de rabia y } \\
\text { agresión. Me sentí tranquilo. }\end{array}$ \\
\hline 8 & $24 \mathrm{~S}$ & $\begin{array}{l}\text { Adele está mirando la televisión con los ojos bien abiertos. Está sentada en una silla de bebe con } \\
\text { algunos juguetes y un cascabel. A momentos pone algunos de estos objetos en su boca. A } \\
\text { momentos ella da vuelta su mirada y me mira a los ojos por unos segundos. En un momento yo } \\
\text { saco una botella de agua de mi mochila y accidentalmente se me cae. En el momento en q la voy } \\
\text { a recoger, escucho que Adele produce un sonido parecido a un grito y yo inmediatamente de } \\
\text { manera instintiva doy vuelta mi cabeza hacia esa dirección. Ella me está mirando con los ojos } \\
\text { abiertos. Le sonrío y continúo agachándome para recoger la botella. Luego de unos instantes, } \\
\text { mira de nuevo en dirección a ella, pero ya no me está mirando, está pegándole a la mesa con un } \\
\text { juguete. Luego da vuelta su cabeza hacia la mesa y pone todo su pulgar adentro de su boca. Se } \\
\text { queda unos minutos variando entre mirar la televisión sin sacar su dedo de su boca. Agita sus } \\
\text { piernas y mueve su cuerpo completo. Luego, comienza a llorar con distress en aumento. Me sentí } \\
\text { culpable porque la conexión entre nosotros se había roto. Me sentí tenso y nervioso. Quería } \\
\text { moverme en la silla. Esperaba que se calmara, que la madre hiciera conexión real con ella, pero } \\
\text { como eso no ocurría sequí tenso física y psíquicamente. }\end{array}$ \\
\hline
\end{tabular}

La Tabla No 2 muestra el registro de la secuencia conductual madre-hija en la observación número 2 acoplado a la secuencia emocional del observador. El observador parece resonar con el estado de Lyn siguiendo la secuencia tranquilidad-incomodidad-subida de arousalimpotencia-rabia-confusión-angustia-acción-resolución-tranquilidad. El observador es

\section{CeIR Vol. 13 (2) - Octubre 2019 ISSN 1988-2939 - www.ceir.info}

(c) Derechos reservados/Copyright de Clínica e investigación Relacional y los autores. Prohibida la reproducción total o parcial sin autorización expresa. Este material es para uso científico y profesional exclusivamente y puede contener información clínica sensible. Los editores no se responsabilizan de los contenidos de los autores. Dirigir las consultas sobre derechos y autorizaciones a ceir@psicoterapiarelacional.es 
capturado (se deja capturar) por la esfera afectiva de la lactante. Presenta una relación diádica conectada emocionalmente.

Tabla $\mathrm{N}^{\circ}$ 2: Registro de la secuencia conductual madre-hija acoplado a la secuencia emocional del observador (Observación $\mathrm{N}_{2}^{2}$ ).

\begin{tabular}{|c|c|c|c|}
\hline Momento & $\begin{array}{l}\text { CONDUCTA } \\
\text { MATERNA }\end{array}$ & CONDUCTA HIJA & $\begin{array}{l}\text { SENSACIONES } \\
\text { OBSERVADOR }\end{array}$ \\
\hline 1 & $\begin{array}{l}\text { Distancia espacial (la } \\
\text { madre está fuera del } \\
\text { campo visual y auditivo } \\
\text { de hija y observador) }\end{array}$ & Despierta y abre los ojos & $\begin{array}{l}\text { Se siente tranquilo, en tercera } \\
\text { persona. Sin estar involucrado } \\
\text { emocionalmente }\end{array}$ \\
\hline 2 & idem & Mueve la cabeza de un lado a otro & Idem \\
\hline 3 & Idem & $\begin{array}{l}\text { Fija mirada en objeto colgante sobre } \\
\text { la cuna }\end{array}$ & Idem \\
\hline 4 & Idem & Agita todo el cuerpo & Se incomoda \\
\hline 5 & Idem & Regurgita & Se activa \\
\hline 6 & Idem & Llora & Siente rabia, impotencia. \\
\hline 7 & idem & Se agita mucho & confusión angustia \\
\hline 8 & idem & Se agita y llora & $\begin{array}{l}\text { Se siente obligado a salir de las } \\
\text { reglas del juego previamente } \\
\text { aceptadas por la madre y busca } \\
\text { su ayuda }\end{array}$ \\
\hline 9 & $\begin{array}{l}\text { Se acerca y se sienta } \\
\text { detrás de la cuna y le } \\
\text { habla }\end{array}$ & Se calma & $\begin{array}{l}\text { Recobra posición de } \\
\text { observador tercera persona }\end{array}$ \\
\hline 10 & & Mira el objeto colgante sobre la cuna & $\begin{array}{l}\text { Curiosidad, contacto con la } \\
\text { madre. Le pregunta que } \\
\text { ocurrió }\end{array}$ \\
\hline 11 & $\begin{array}{l}\text { Madre responde que no } \\
\text { se lo explica ya que } \\
\text { comió y durmió. }\end{array}$ & & apertura \\
\hline
\end{tabular}

La tabla No 3 muestra los registros del devenir emocional regulatorio de la hija y la transferencia/ contratransferencia del observador según ausencia/ presencia, materna. 
Tabla $N^{\circ} 3$ Registros del devenir emocional regulatorio de la hija y transferencia/ contratransferencia del observador según ausencia/ presencia, materna. Observación $\mathrm{N}^{\circ} 2$.

\begin{tabular}{|c|c|c|c|}
\hline Momento & SIGNO MADRE & SIGNO HIJA & $\begin{array}{l}\text { TRANSFERENCIA/ } \\
\text { CONTRATRANSFERENCIA } \\
\text { DEL OBSERVADOR }\end{array}$ \\
\hline $1-2$ & $\begin{array}{l}\text { Ausencia en el campo } \\
\text { vincular }\end{array}$ & $\begin{array}{lll}\text { Regulación } & \text { emocional } & \text { del } \\
\text { despertar } & & \end{array}$ & Regulado, cómodo. \\
\hline 3 & Idem & $\begin{array}{l}\text { Intento de regulación } \\
\text { Con conexión a objeto familiar }\end{array}$ & \\
\hline 4,5 & Idem & $\begin{array}{l}\text { Falla en la regulación emocional y } \\
\text { caída al síntoma somático }\end{array}$ & Incomodidad y duda \\
\hline 6 & Idem & Llama por ayuda & tensión \\
\hline 7 & & Desorganización & $\begin{array}{l}\text { Aumenta intensidad de } \\
\text { tensión }\end{array}$ \\
\hline 8 & & $\begin{array}{l}\text { Intensa desorganización: } \\
\text { Agitación psicomotora }\end{array}$ & $\begin{array}{l}\text { Aumento en la intensidad de } \\
\text { afectos negativos. } \\
\text { Necesidad e impulso para } \\
\text { actuar }\end{array}$ \\
\hline 9 & $\begin{array}{l}\text { Presencia en el } \\
\text { campo vincular }\end{array}$ & Se Calma & Comodidad y curiosidad. \\
\hline 10 & $\begin{array}{l}\text { Madre no encuentra } \\
\text { la causa }\end{array}$ & Se autoregula & Calma \\
\hline
\end{tabular}

La tabla No 3 muestra la relación de los tres sujetos participantes: madre-observadorlactante en sus resultados de regulación. El lactante despierta regulado, en bienestar. La madre está ausente y el observador cómodo y regulado. Luego el lactante en un intento de regulación fija su mirada en objeto familiar y aún sigue en bienestar. La madre está ausente. La lactante se incomoda y aparece un proceso somático para mostrarlo: regurgita. El observador se incomoda y duda si ir a buscar a la madre (debiera salir de la consigna que le prohíbe intervenir. Siente que aumenta la tensión y la lactante comienza a desorganizar su conducta. Aumenta la tensión de ambos. La lactante se desorganiza aún más, tanto que llega a la agitación psicomotora. El observador actúa (llama a la madre). Llega la madre, se calma la lactante y se calma el observador. La presencia materna es un potente regulador emocional. La desorganización del lactante es un potente activador del observador forzándolo a trasgredir el acuerdo normativo de la observación. El instrumento con el cual 
el observador mide los estados afectivos de la lactante es su propio sentir. Y por esto queda consignado.

La Tabla No 4 muestra los conceptos dinámicos seleccionados como referentes del proceso de significación en: a. Dos dimensiones: proceso y estructura. B. Seis términos:1. Conductas 2. afectos (o emociones) positivas (ej. alegría y exploración), negativas (ej. Rabia, malestar) o primitivos(confusos) 3. Procesos somáticos o psíquicos; 4. regulaciones: dinámicas o permanentes .5. límites: las reglas del sistema medidas por el arousal de los miembros incluido el observador -el arousal entendido como: el tono afectivo de las interacciones que el observador captura por transferencia y contratransferencia. Este tono puede ser excitado en positivo o negativo o hipo excitado con: desvitalización, hiponimia, hipoactividad y tendencia al sopor y la somnolencia .6 sistema resultante: la integración de todos los anteriores.

Tabla $\mathrm{N}_{4}$ : Conceptualización Teórica de las dinámicas presentes en el Campo Vincular.

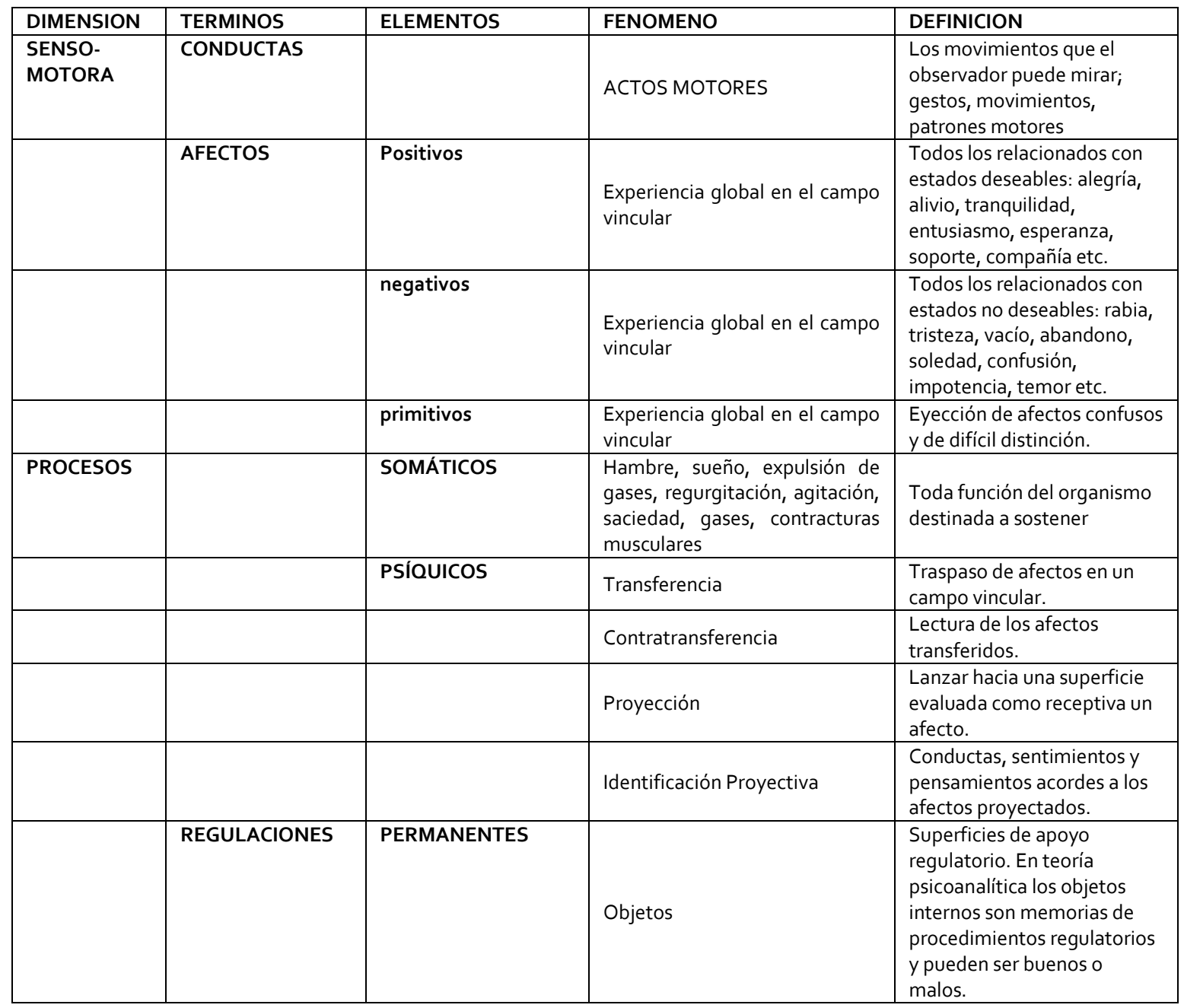




\begin{tabular}{|l|l|l|l|l|}
\hline & & DINÁMICAS & & $\begin{array}{l}\text { Procedimiento utilizado para } \\
\text { regular la intensidad de los } \\
\text { afectos . La defensa de más } \\
\text { baja elaboración es dividir la } \\
\text { vivencia y expulsar lo } \\
\text { negativo fuera del sistema. } \\
\text { Esto puede ser como actos } \\
\text { corporales o proyectándola } \\
\text { en el campo vincular a un } \\
\text { receptor disponible. Las } \\
\text { defensas altas, elaboran los } \\
\text { afectos negativos } \\
\text { tolerándolos, } \\
\text { resignificándolos o } \\
\text { transformándolos. }\end{array}$ \\
\hline & LIMITES & Defensa & & Excitabilidad del sistema \\
\hline TURA & SISTEMA & UMBRALES & Arousal & $\begin{array}{l}\text { Negociación de reglas de } \\
\text { ajuste para la sintonía entre } \\
\text { al menos dos seres vivos que } \\
\text { conforman un sistema }\end{array}$ \\
\hline
\end{tabular}

Se definen y describen los fenómenos en las dos columnas siguientes.

En la figura $\mathrm{N}^{\circ} 1$ se muestra el modelo del campo vincular observacional integrando los conceptos dinámicos seleccionados. El modelo permite organizar la observación y producir sentido clínico.

Figura $\mathrm{N}^{\circ} 1$ Dimensiones del campo vincular observacional que dan como resultado el arousal y sus limites

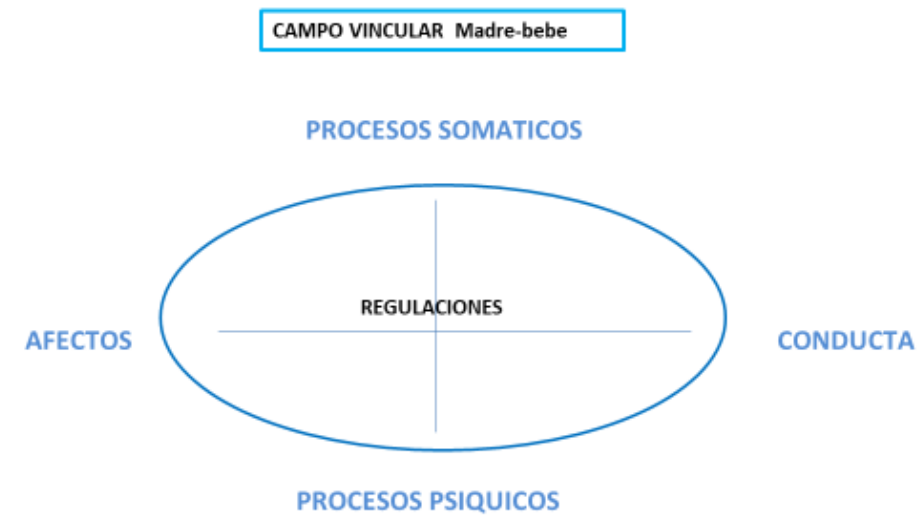

En la Tabla $N^{\circ} 5$ se muestra un modo de leer el Campo Vincular observacional a través de las dimensiones miradas y sentidas por el observador.

\section{CeIR Vol. 13 (2) - Octubre 2019 ISSN 1988-2939 - www.ceir.info}

(c) Derechos reservados/Copyright de Clínica e investigación Relacional y los autores. Prohibida la reproducción total o parcial sin autorización expresa. Este material es para uso científico y profesional exclusivamente y puede contener información clínica sensible. Los editores no se responsabilizan de los contenidos de los autores. Dirigir las consultas sobre derechos y autorizaciones a ceir@psicoterapiarelacional.es 
Tabla $\mathrm{N}^{\circ} 5$ Lectura del Campo Vincular observacional a través de las dimensiones miradas y sentidas por el observador. Instrumento de observación que discrimina funcionamiento del Campo Vincular observacional

\begin{tabular}{|l|l|l|l|}
\hline DIMENSION & RIESGO & SUFICIENTE & OPTIMO \\
\hline AFECTO & Negativo & Neutro & Positivo \\
\hline CONDUCTA & Desorganizada & intermedia & Organizada \\
\hline AROUSAL & Activado & Neutro & \\
\hline & Desactivado & & \\
\hline $\begin{array}{l}\text { PROCESOS } \\
\text { SOMATICOS }\end{array}$ & Patología & Manifestaciones & \\
\hline & Síntomas & & \\
\hline
\end{tabular}

Para el observador las dimensiones observables (Tabla No 5 ) son:

-el afecto: negativo, neutros o positivos;

-los procesos físicos a través de manifestaciones somáticas, síntomas o patologías establecidas;

- las conductas: organizadas que presentan estructura de inicio, desarrollo y fin o las desorganizadas donde hay actividad sin dirección ni foco.

Si aplicamos este cuadro a la observación numero dos veríamos: el afecto oscila entre negativo y neutro. La conducta entre organizada y desorganizada. El arousal va de activado a neutro. $Y$ los procesos somáticos presentan manifestaciones (regurgitación) sin ser síntomas permanentes ni patología. Se puede concluir que el sistema tiene flexibilidad y por lo mismo se encuentra en adaptación; que hay una tendencia a afectos negativos ya presentar el arousal activado llegando a la desorganización de la conducta y manifestaciones somáticas. A pesar de no haber patología instalada en el lactante se trata de un campo vincular observacional con un discreto riesgo expresado por manifestaciones en el soma y el tono de arousal. En caso de evaluar o intervenir clínicamente habría que chequear la ansiedad y el ánimo maternos.

\section{DISCUSIÓN Y CONCLUSIONES}

Crear significado relacional requiere de un proceso de construcción de sentido. Por lo mismo un recorrido que organiza la información hasta hacerla significar termina en un modelo, en un procedimiento de reflexión que podremos repetir cada vez que sea 
necesario utilizar. Los modelos de comprensión son formas de organizar la información dotándola de sentido. El proceso de construcción del modelo presentado en este articulo inicia su recorrido en la observación interaccional realizada por un observador participante, y sigue con la articulación de los conceptos dinámicos seleccionados.

Se ha utilizado una de las observaciones (la numero dos) para mostrar el recorrido de sentido hasta llegar al modelo de comprensión que podrá ser utilizado en las distintas observaciones.

Los conceptos cumplen con la función de transformar la cualidad de la observación. La asignación de significado, aún si transitorio, direcciona las observaciones siguientes hacia la construcción de un sentido global. Sentidos y significados varían en el tiempo en conjunto con el desarrollo del lactante observado.

En esta propuesta se han organizado los referentes en sus dimensiones sintácticas y semánticas. (Greimas, 1973).

En la dimensión sintáctica no solo se dispone una secuencia temporal que ordena sujetos y predicados, causas y efectos, sino que cada acontecimiento registrado es como un nudo que aglutina diversos significados. Estos nudos significantes dispuestos en la línea temporal (horizontal) se han nominado como: afectos, conductas, procesos somáticos o psíquicos. La secuencia de estos acontecimientos relacionales permite crear un frame, un trozo interaccional desde el cual es posible recoger su sentido.

En la dimensión semántica la articulación de significado en cambio es vertical. Se ordena articulando el acontecimiento a un referente conceptual dinámico. La selección de los referentes utilizados se realizó en función del uso corriente que se hace en psicología de los mismos al referirse a comunicación humana (tabla $\mathrm{N}_{4}$ ). De este modo el frame queda cargado de un orden sensible temporal (conductas, procesos somáticos, afectos) y de otro pensable (referencial) que da cuenta de los procesos psíquicos. El orden sensible, el que se observa o siente (recogido por resonancias o transferencia) es aportado por el observador a través de sus registros en el momento mismo que ocurren los hechos (tiempo cero). El orden pensable es un tiempo dos del observador (Cordella, 2009) que ocurre luego que el observador enlaza con los referentes en el tiempo uno.

El orden pensable a su vez se ha organizado en dos dimensiones: lo estructural y lo procesal. Esta distinción permite ordenar lo permanente, de lo cambiante. La estructura es la repetición constante del mismo proceso a pesar de los cambios ambientales y representa meta niveles de funcionamiento como son por el ejemplo el arousal . Lo procesal, en cambio, es el afán adaptativo, las formas de defensa a los impactos del ambiente, los 
"mecanismos" de defensa. Es la repetición constante de estas adaptaciones que al incorporarse se automatiza, se hace estructura.

Los mecanismos de defensa son procesos psíquicos, no evidentes a la primera observación sensible. Por esto, el acceso a los procesos psíquicos como son la: Transferencia, Contratransferencia, Proyección e Identificación Proyectiva requiere de un segundo tiempo. Un tiempo reflexivo, un observador reflexivo que haga inferencias: deducciones, inducciones y abducciones (Pierce, 1931).

Realizadas las operaciones de construcción de sentido es posible presentar un modelo donde disponer los elementos sensibles y reflexivos de modo de hacerlos significar para la clínica.

En el Modelo que se presenta se han incluido cuatro dimensiones. Dispuestas en vectores tensivos. Un eje vertical y otro horizontal, cada uno con dos polos de una dimensión y que conforman cuatro cuadrantes. En la vertical, los procesos: somático y psíquicos. En la horizontal los afectivos y conductuales. Las dinámicas entre estas dimensiones, es decir las tensiones que se generan en el operar de estas cuatro dimensiones, son el sistema, lo actualizan, lo reproducen. Este sistema funciona con un tono, el arousal que le facilita o restringe las mismas cuatro dimensiones y propone formas de funcionamiento y por lo mismo lo configura. Se trata de un modelo en el cual cada parte participa y construye un todo que modula a su vez cada parte que podríamos llamar "Campo Vincular Observacional"

Kurt Lewin emplea la noción de campo para describir el desarrollo y la conducta del niño como resultado de la organización de fuerzas en una relación. La fuerza es definida por su dirección, intensidad y punto de aplicación ( (Matuk, 2018).

El Campo Vincular es como un sistema auto-regulado creado a través del aprendizaje de modos- de- estar- con (Stern, 1987) durante el desarrollo que opera como regulador interaccional, emocional y somático.

La semiología de este campo vincular requiere de un observador sensible, capaz de percibir con todos sus sentidos lo que allí ocurre.

Se ha dado importancia al Observador y a los procesos mentales que debe hacer para constituirse como Observador eficiente, capaz de comunicarse ampliando su receptividad perceptiva, empática e inconsciente (Izzedin- Bouquet, 2009). para poder distinguir el funcionamiento del CV.

El Observador está obligado a regresar a sus etapas tempranas para empatizar con las vivencias del lactante y luego a progresar hacia la comprensión adulta, el incremento de su 
darse cuenta y el manejo de conceptos que lo faciliten parecen ser deseables en la formación de los profesionales que trabajan con diadas madre-hijo/a.

Estos afectos son trasferidos al observador vía sintonía (Cordella, 2009) neuronas espejo y procesos empáticos. (Rizzolatti \& Craighero, 2004; Green, 2018). El concepto de transferencia, hay que notar, se usa más cuando se trata de afectos positivos, mientras que la proyección en los negativos (Habibi-Kohlen, 2018).

El proceso de aprendizaje que realiza el observador lo va transformando en un observador sensible y reflexivo capaz de involucrarse integralmente. Así participa con su psiquis y su cuerpo de la experiencia del campo vincular en observación. El cuerpo es parte integral de la psiquis (Fonagy, Gergerly,Jurist \& Target , 2002) así como la psiquis del cuerpo. Se aprende desde la experiencia, no sobre la experiencia. el observador comprende que él está implicado y quedará incluido en lo observado (Maturana, 1995). Todo fenómeno observado contiene al observador (Vidal, 2019).

Este aprendizaje le permite inaugurar un estado de conciencia vacío de "poder/querer/deber/saber" desde el cual dar espacio al conocer.

La $O B$ es una metodología que utiliza tanto la primera como la tercera persona en su análisis. El Observador queda incluido en lo observado (Maturana, 1995) Como lo describe la segunda cibernética, todo fenómeno observado contiene al Observador (Vidal, 2019) y el observador pasa a ser un resonador afectivo. (Holmes et al., 2019) (Cordella, 2009).

En el ejemplo de Adele vemos que estas defensas se comienzan a instalar desde la actividad corporal. Adele tiene solo 10 meses cuando ya intenta regularse con objetos concretos. Ya que no lo consigue recurre a expulsar desde el cuerpo la incomodidad. Como es muy pequeña, solo puede pedir ayuda con acciones motoras que se incrementan hasta la agitación si no consigue solución contextual. Lo interesantes es que el Observador logra capturar afectos negativos condensados en la lactante y los puede descifrar. La intensidad logra incomodarlo a tal punto que lo eyecta fuera de su rol, aun estando preocupado de conservarlo. Para que esto ocurra, el Observador evalúa una intensidad tal que está poniendo en riesgo algo fundamental para el desarrollo del lactante. Estos traumas cotidianos se van acumulando y pueden requerir defensas al dolor físico o psíquico que producen. La disociación somato-psiquica pasa a ser una posición no sólo preferencial sino necesaria para sobrevivir. (Farina, B., Liotti, M., \& Imperatori, C., 2019).

Otras formas de observar las relaciones madre-bebe se basan en el consenso de jueces y en la repetición de escenas previamente grabadas. Tal es el caso de las Pautas de observación. Por ejemplo: 
a) The Attachment Q Sort -Observer version (AOS; Waters \& Deane, 1985)

b) The Strange Situation Procedure (Ainsworth, M. D. S., Blehar, M. C., Waters, E., \& Wall, S. N. (2015). Patterns of attachment: A psychological study of the strange situation. Psychology Press. (SSP; Ainsworth et al., 1979).

c) Maternal Behavior Q-Sort (MBOS) to code sensitivity in dyadic observations (Pederson et al., 1990). Estas se usan con fines de investigación y requieren de certificación. Es decir, se somete a un entrenamiento de codificación a los investigadores como una forma de estandarizar la observación y hacerla significar en la comparación con otras observaciones. Este modo de acceder a los fenómenos interaccionales se enfoca más en crear verdad a través del tamiz matemático estadístico sobre poblaciones que en la veracidad que busca legitimar el particular de una interacción. Uno apunta más a la naturaleza humana compartida por la especie, en un intento de objetivación. Mientras este otro, a las diferencias individuales dentro de la misma especie, en un intento de acceder a lo subjetivo.

Para los fines psicoterapéuticos ambas miradas son necesarias para la comprensión global. Sin embargo, solo la observación de una interacción es un trozo de realidad que puede aportar información si la hacemos significar con referentes consensuados.

El modelo del campo vincular observacional permite comprender el funcionamiento tanto de una relación como de tramos interaccionales, con mayor o menor detalle dependiendo del entrenamiento del observador ya que todo fenómeno observado contiene al observador (Vidal, 2019).

La tarea de conformar un Campo Vincular desde el registro de OB se afina si el observador es entrenado en su capacidad de observación y auto-observación. Este sistema, comprendido desde la semiología relacional incluye:

a. Lo observable: conducta; procesos somáticos.

b. Lo sensible: afectos (recogidos a través de resonancia o transferencia).

c. Lo interpretable: procesos psíquicos d. El resultado: Arousal y Límites.

La descripción y evaluación del Campo Vincular facilitaría reconocer las diadas disfuncionales y los puntos de acceso sensibles al cambio en las futuras intervenciones.

En resumen, se presenta un modelo de generación de sentido que, partiendo por los registros de una $O B$ construye categorías de la observación de conductas, afectos, procesos somáticos y psíquicos que en conjunto conforman campos vinculares los que muestran sus límites y arousal de funcionamiento. El modelo de campo vincular observacional es útil en la clínica de relaciones diádicas en especial las del desarrollo infantil. 


\section{REFERENCIAS}

1. Aguayo, J., \& Salomonsson, B. (2017). The Study and Treatment of Mothers and Infants, Then and Now: Melanie Klein's "Notes on Baby" in a Contemporary Psychoanalytic Context. The Psychoanalytic Quarterly, 86(2), 383-408.

2. Ainsworth, M. S. (1979). Infant-mother attachment. American psychologist, 34(10), 932.

3. Beebe, B., Jaffe, J., Markese, S., Buck, K., Chen, H., Cohen, P., Bahrick, L., Andrews, H., \& Feldstein, S. (2010). The origins of 12-month attachment: A microanalysis of 4-month mother-infant interaction. Attachment \& Human Development 12:3-141.

4. Bick, E. (1964). Notes on infant observation in psycho-analytic training. International Journal of Psycho-Analysis, 45, 558-566.

5. Bion WR. (1982). La Tabla y la Cesura. Gedisa, Barcelona.

6. Bion, W. R. (1984). Learning from experience (1962). London: Karnac.

7. Cordella P. (2009). Campos de sintonía somática durante el desarrollo infantil. Revista Chilena de pediatría, 80(5), 407-419.

8. Cordella, P. (2014). Qué es semiosis. Revista Gaceta de Psiquiatría Universitaria, 10, 390394.

9. Eco, U ( 1988) Signo Editorial Labor, Barcelona.

10. Farina, B., Liotti, M., \& Imperatori, C. (2019). The role of attachment trauma and disintegrative pathogenic processes in the traumatic-dissociative dimension. Frontiers in psychology, 10, 933.

11. Fonagy P, Gergely G, Jurist E \& Target M. (2002). Affect Regulation, Mentalization, and the Development of the Self. Karnac, London.

12. Fonagy, P. (2018). Affect regulation, mentalization and the development of the self. Routledge, London

13. Fonagy, P., \& Allison, E. (2012). What is mentalization? The concept and its foundations in developmental research. In Minding the child: Mentalization-based interventions with children, young people and their families, pp. 11-34. Routledge, London.

14. Freud, A. (1958). Child observation and prediction of development: A memorial lecture in honor of Ernst Kris. The psychoanalytic study of the child, 13(1), 92-116.

15. Freud, S. (1905). Tres ensayos de teoría sexual y otras obras. Obras completas, 7, 109-211.

16. Freud, W. E. (1976). Mother-infant observation in the teaching of psychoanalysis. Psyche (Stuttg), 30(8), 723-743

17. Green, A. (2000). Science and science fiction in infant research. Clinical and observational psychoanalytic research: Roots of a controversy, 41-72.

18. Greimas, AJ. (1973). En torno al sentido: ensayos semióticos. Fragua, Madrid.

19. Habibi-Kohlen, D. (2018). Paths of the countertransference in the analyst-clinical examples of working through. The International Journal of Psychoanalysis, 99(2), 391-410. 
20. Hofer, M. A. (1984). Relationships as regulators: A psychobiologic perspective on bereavement. Psychosomatic Medicine, 46(3), 183-197

21. Holmes, J., Adams, S., Aguayo, J., Aron, L., Aron, L., Avdi, E., ... \& Guthrie, E. (2019). The use of an object. In A Practical Psychoanalytic Guide to Reflexive Research: The Reverie Research Method (Vol. 2, No. 2, p.p 1-9). Philadelphia: Tavistock Publication.

22. Hutchon, B., Gibbs, D., Harniess, P., Jary, S., Crossley, S. L., Moffat, J. V.,\& Basu, A. P. (2019). Early intervention programmes for infants at high risk of atypical neurodevelopmental outcome. Developmental Medicine \& Child Neurology, Advance Online Publication.

23. Ismail, F. Y., Fatemi, A., \& Johnston, M. V. (2017). Cerebral plasticity: windows of opportunity in the developing brain. European Journal of Paediatric Neurology, 21(1), 2348.

24. Izzedin-Bouquet, R. (2009). El método de observación de bebés de Esther Bick. Perinatología y Reproducción Humana, 23(4), 219-222.

25. Klein, M. (1952). On observing the behavior of young infants. In The Writings of Melanie Klein, Vol. 3. Hogarth, London. pp. 94-121

26. Matuk, G. D. (2018). Constelación y campo: psicología de Kurt Lewin. Universidad Autónoma del Estado de Morelos.

27. Maturana HR. (1995). La realidad: objetiva o construida? II. Fundamentos biológicos del conocimiento. Anthropos, Barcelona.

28. Minjollet, P., Garez, V., Héroux, C., Letamendia, E., \& Apter, G. Élaboration d'une échelle d'observation des nouveau-nés en maternité. Soins Pédiatrie/Puériculture, 39(301), 42-47.

29. Ng, L., Bampton, C., Stevens, S., \& Woods, P. (2017). The infant observation task as a tool in psychiatric practice. Australasian Psychiatry, 25(3), 236-238.

30. Pederson, G. Moran, C. Sitko, K. Campbell, K. Ghesquire, H. ActonMaternal sensitivity and the security of infant-mother attachment: A Q-sort study Child Development, 61 (6) (1990), pp. 1974-1983, 10.2307/1130851.

31. Peirce, C. S. (1931). Collected papers of charles sanders peirce. Harvard University Press.

32. Perry, B. D., Pollard, R. A., Blakley, T. L., Baker, W. L., \& Vigilante, D. (1995). Childhood trauma, the neurobiology of adaptation, and "use-dependent" development of the brain: How "states" become "traits". Infant mental health journal, 16(4), 271-291.

33. Pratt, M., Apter-Levi, Y., Vakart, A., Kanat-Maymon, Y., Zagoory-Sharon, O., \& Feldman, R. (2017). Mother-child adrenocortical synchrony: Moderation by dyadic relational behavior. Hormones and Behavior, 89, 167-175. https://doi.

org/10.1016/j.yhbeh.2017.01.003

34. Riva, G. (2018). The neuroscience of body memory: From the self through the space to the others. Cortex, 104, 241-260.

35. Rizzolatti, G., \& Craighero, L. (2004). The mirror-neuron system. Annu. Rev. Neurosci., 27, 169-192. 
36. Rustin, M. (2014). The relevance of infant observation for early intervention: containment in theory and practice. Infant Observation, 17(2), 97-114.

37. Schore, J. R., \& Schore, A. N. (2008). Modern attachment theory: The central role of affect regulation in development and treatment. Clinical social work journal, 36(1), 9-20.

38. Sravanti, L. (2017). Nurture the nature. Indian Journal of Psychiatry, 59(3), 385.

39. Stern, D. N. (1997). La constelación maternal: un enfoque unificado de la psicoterapia con padres e hijos. Paidós Ibérica

40. Symington, J. (2018). Mrs Bick and infant observation. In Surviving space (pp. 105-116). Routledge, London.

41. Taylor, G. J. (2018). Symbolism, symbolization, and trauma in psychosomatic theory. In Psychosomatics Today (pp. 181-199). Routledge, London

42. Tost, H., Champagne, F. A., \& Meyer-Lindenberg, A. (2015). Environmental influence in the brain, human welfare and mental health. Nature Neuroscience, 18(10), 1421-1431. https://doi.org/10.1038/nn.4108

43. Tottenham, N. (2015). Social scaffolding of human amygdala-mPFCcircuit development. Society for Neuroscience, 10(5), 489-499. https://doi.org/10.1080/17470 919.2015.1087424

44. Vidal, J. P. (2019). Perspectivas de La Teoría Sistémica para la Observación de la Complejidad. Complexitas-Revista de Filosofia Temática, 2(2), 95-108.

45. Waters, E., \& Deane, K. E. (1985). Defining and assessing individual differences in attachment relationships: Q-methodology and the organization of behavior in infancy and early childhood. Monographs of the society for research in child development, 50(1-2), 41-65.;

46. Winnicott, DW. (1993). Los procesos de maduración y el ambiente facilitador: estudios para una teoría del desarrollo emocional. Paidós, Buenos Aires.

47. Winnicott, D. (1962). La integración del yo en el desarrollo del niño. D. Winnicott (1993). Los procesos de maduración y el ambiente facilitador. Buenos Aires: Paidós.

48. Winnicott, D. W. (1941). The observation of infants in a set situation. International Journal of Psycho-Analysis, 22, 229-249.

49. Zaphiriou Woods, M., \& Pretorius, I. M. (2016). Observing, playing and supporting development: Anna Freud's toddler groups past and present. Journal of Child Psychotherapy, 42(2), 135-151.

Original recibido con fecha: 28/8/2019 Revisado: 30/9/2019 Aceptado: 30/09/2019 